\section{Fetal Diagnosis and Therapy}

Barini, R. 71

Bennini, J.R. 71

Brady, P.D. 25

Cannie, M. 80

Castañón, M. 101

Chiu, P. 40

Claus, F. 80, 88

Cohen-Overbeek, T.E. 55

Costa, F. 111

Cruz-Martinez, R. 18, 88, 101

da Silva, M.M. 64

de Fátima Assunção Braga, A. 71

De Keyzer, F. 80

Decraene, J. 88

DeKoninck, P. 80,88

Deprest, J.A. 6, 18, 25, 80, 88, 101

Devlieger, R. 88

Devriendt, K. 25

Done, E. 18, 80, 101

Duarte, S.A. 64

Fink, A.M. 111

Fryns, J.-P. 25

Gratacós, E. 5, 6, 18, 88, 101

Gucciardo, L. 80,88
Haino, K. 108

Harrison, M.R. 78

Hernández-Andrade, E. 18, 101

Hirayama, Y. 108

Hop, W. 55

Itsukaichi, M. 108

Jani, J. 80

Kaganov, H. 111

Kikuchi, A. 108

Kim, P.C.W. 40

Lally, K.P. 46

Machado Rosa, I.R. 71

Mah, V.K. 40

Martínez, J.M. 101

Michielsen, K. 88

Moreno-Alvarez, O. 18, 88, 101

Ng, J. 111

Nicolaides, K. 6

Numata, M. 108

O’Mahony, E. 111

Palma-Dias, R. 111

Peralta, C.F.A. 71

Pimenta, E.J.d.A. 64

Reiss, I. 55
Richter, J. 88

Ruano, R. 64

Sakakibara, S. 108

Sampaio Rousselet, M. 71

Sandaite, I. 80, 88

Sbragia, L. 71

Schaible, T. 55

Serikawa, T. 108

Siemer, J. 55

Srisupundit, K. 25

Takakuwa, K. 108

Takashi, E. 64

Tanaka, K. 108

Tannuri, U. 64

Tibboel, D. 55

Tsao, K. 46

van de Ven, K. 55

van den Hout, L. 55

Van Mieghem, T. 80, 88

Vermeesch, J.R. 25

Wessel, L. 55

Zugaib, M. 64

\title{
Subject Index Vol. 29, No. 1, 2011
}

Bronchopulmonary dysplasia 55

- sequestration 111

Congenital abnormalities 111

- diaphragmatic hernia $6,18,25,40,46$, $55,64,71,80,88,101,108$

Cystic adenomatoid malformation of lung 111

Diaphragm 25

Diaphragmatic hernia 71

Doppler 18

Endoscopic tracheal occlusion, fetal 6

Endotracheal occlusion 71

Extracorporeal membrane oxygenation 46, 55

Fetal endoscopic tracheal occlusion 64

- intervention 6
Fetoscopic endoluminal tracheal occlusion 88

- tracheal occlusion 18

Fetoscopy 6, 64, 71

Fractional moving blood volume 101

Genetics 25

Hernia, diaphragmatic 111

Herniation 71

Hidden mortality 40

Institution-based research 40

Intrapulmonary circulation 18

Liver herniation 64

Lung 25

- expansion 71

- perfusion 18,101

- surgery 64

- volume 80
Lung-to-head ratio 55, 80, 101

Magnetic resonance imaging 80, 88, 111

Mesenchymal cell function 25

Minimally invasive surgery 46

Morgagni hernia 108

Pericardial effusion 108

Population-based research 40

Prenatal diagnosis 80, 108

- imaging 88

Preoperative stabilization 46

Pulmonary hypoplasia $6,64,80,88$

Retinoic acid 25

Retinoid signalling 25

Survival rates 55

Tracheal occlusion 71, 101

Ultrasonography, prenatal 111

Ultrasound 88

(C) 2011 S. Karger AG, Basel 